\title{
A 5-Year Retrospective Analysis of Raoultella planticola Bacteriuria
}

\author{
Sai Vikram Alampoondi \\ Venkataramanan \\ Lovin George \\ Kamal Kant Sahu (D) \\ George M Abraham (ID)
}

Department of Medicine, Saint Vincent Hospital, Worcester, MA, 01608, USA
Correspondence: Kamal Kant Sahu Department of Medicine, Saint Vincent Hospital, Worcester, MA, 01608, USA Email drkksahu85@gmail.com
Introduction: Raoultella planticola is an aerobic gram-negative rod predominantly found in soil and aquatic environments. The typical reservoirs of Raoultella spp. include the gastrointestinal tract and the upper respiratory tract. It usually causes pneumonia, biliary tract infections, and bacteremia. Urinary tract infection (UTI) secondary to R. planticola is an uncommon entity. Less than 10 cases of $R$. planticola-associated UTIs in adults have been published in the literature to date.

Objective: This is a single institution retrospective study undertaken to identify the epidemiology, patient characteristics, clinical spectrum, predisposing risk factors and the outcome of patients with UTI caused by $R$. planticola.

Results: A total of $37 \mathrm{R}$. planticola isolates were identified in urine samples over a 5-year study period. The mean age of the patient population was 77 years. The most common comorbidity was diabetes mellitus, which was present in 16 patients. Only 3 patients had a history of steroid use, an immunosuppressive condition, or were on chemotherapy. The most common presenting complaint was altered mental status followed by fever. Resistance to ampicillin was found in 35 isolates which seems to be an intrinsic characteristic of Raoultella spp. and 2 isolates were multidrug-resistant, but still susceptible to ciprofloxacin. The average length of stay was 3 days, and the average duration of antibiotic administration was 8 days. Ciprofloxacin was the most frequently prescribed antibiotic ( 9 patients). The severity of infection ranged from simple cystitis in 15 patients to urosepsis in 2 patients and septic shock in 2 patients. There were no mortalities in our cohort.

Conclusion: Our study revealed that patients with $R$. planticola UTI had higher proportion of diabetes mellitus, renal failure compared to the general population. Our study also confirms the intrinsic resistance to ampicillin of Raoultella spp., which has been documented previously in the literature.

Keywords: infection, antibiotics, Raoultella, urinary tract infection, bacteriuria

\section{Introduction}

Infections related to rare pathogens are very challenging to treat due to lack of enough data in the medical literature. ${ }^{1,2}$ Raoultella planticola is a ubiquitous, non-motile, aerobic gram-negative bacteria. It belongs to the genus Raoultella under the Enterobacteriaceae family. Two species are clinically important: [1] Raoultella planticola and [2] Raoultella ornithinolytica. The microbiological identification of $R$. planticola remains a challenge even after 30 years of its identification. ${ }^{3,4}$ This is because the conventional phenotypic assessments often misidentify it as Klebsiella spp., which is its remarkably close relative in the Enterobacteriaceae family. This might be one of the many reasons that the incidence of infections caused by $R$. planticola is underreported. 
The gastrointestinal tract and the upper respiratory tract are the typical reservoirs of $R$. planticola. ${ }^{5}$ It commonly causes pneumonia, biliary tract infections, and bacteremia. ${ }^{6-9}$ Only a handful of cases of urinary tract infection (UTI) caused by $R$. planticola in adults have thus far been published in the literature. ${ }^{10-16}$ Most of the UTIs have been reported in immunocompromised adults, in patients with urinary tract procedures or instrumentation. ${ }^{11-13,15}$

The recent emergence of extended-spectrum $\beta$ lactamase (ESBL) producing and carbapenem-resistant nosocomial $R$. planticola infections is a matter of concern. These strains can act as a reservoir for these resistance genes. ${ }^{17}$

Except for a few case reports/series, there has been no published comprehensive review or original study on $R$. planticola-associated UTIs. We conducted a retrospective study to better understand the characteristics of the organism, risk factors for acquisition of infection and to analyze the outcomes associated with UTI caused by $R$. planticola.

\section{Materials and Methods Search Strategy}

This was a retrospective study conducted at a communitybased teaching hospital in Massachusetts, United States. The study included data from January 2015 to September 2020 and included all urine cultures positive for $R$. planticola at our institution. The study was approved by our institutional review board (IRB \#2020 -142).

\section{Selection and Inclusion Criteria}

All adult patients ( $>18$ years) who had a urine culture growing R. planticola were included in the study. Primary identification and sensitivities of colonies were done with VITEK ${ }^{\circledR} 2$ compact automated system and confirmation was done with API 20-E, both from BioMérieux.

\section{Data Extraction}

Records of selected patients were reviewed and information including the demographics, clinical symptoms, physical examination findings, laboratory data, radiological parameters and microbiological data were extracted. Further details on hospitalization including the severity of the illness, antibiotics administered, hospital course and outcomes were also obtained. The data extracted was verified by two independent researchers. The data was then entered into a spreadsheet.

\section{Data Analysis}

All categorical data were expressed as counts, whereas continuous data were expressed as a mean. The data was analyzed using Google sheets ${ }^{\circledR}$. No statistical analysis was performed given the small sample size.

\section{Results}

\section{Demographic Details}

A total of 37 patients had a positive urine culture for R. planticola. The demographics of the patients are listed in Table 1 . The mean age of our cohort was 77 years ( 47 years 91 years). There were 28 females and 9 males in total. Out of our 37 patients, 9 were residents of nursing homes or group homes, and 28 presented from home. The body mass index (BMI) was measured to be $<19 \mathrm{~kg} / \mathrm{m}^{2}$ in 3 patients, between $19 \mathrm{~kg} / \mathrm{m}^{2}$ to $25 \mathrm{~kg} / \mathrm{m}^{2}$ in 15 patients, between $25 \mathrm{~kg} / \mathrm{m}^{2}$ to $30 \mathrm{~kg} / \mathrm{m}^{2}$ in 10 patients and more than $30 \mathrm{~kg} / \mathrm{m}^{2}$ in 9 patients. The mean BMI was $26.64 \mathrm{~kg} / \mathrm{m}^{2}$.

\section{Risk Factors}

We studied the systemic and genitourinary factors in our population that were associated and/or have prognostic significance to our patients with $R$. planticola-associated UTIs (Table 1).

\section{Systemic Characteristics}

Alcohol consumption was reported by 5 patients while 32 patients denied it. None of the patients was active smokers with 17 endorsing prior tobacco use. Amongst the 37 patients, 16 patients had a history of diabetes mellitus. Liver dysfunction was present in 7 patients. Chronic corticosteroid and immunosuppressant use were reported by 1 patient each, and chemotherapy by 2 patients.

\section{Genitourinary Characteristics}

A history of chronic kidney disease was present in 18 patients. Out of these 18 patients, 5 had an acute on chronic kidney injury. There was a history of a urological procedure or genitourinary instrumentation in 4 patients, with 2 having the procedure within a month of their UTI. An underlying structural abnormality of the genitourinary tract was present in 9 patients as depicted in Figure 1. A history of renal transplantation was present in 1 patient. 


\begin{tabular}{|c|c|c|c|c|c|c|c|c|c|c|c|c|c|c|c|c|c|c|c|c|c|}
\hline 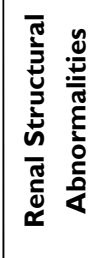 & $\begin{array}{l}0 \\
\stackrel{0}{0} \\
z\end{array}$ & $\stackrel{0}{\tilde{0}}$ & $\begin{array}{l}0 \\
\tilde{0} \\
z\end{array}$ & $\begin{array}{l}0 \\
\check{0} \\
z\end{array}$ & $\stackrel{0}{0}$ & $\begin{array}{l}\text { o } \\
\text { zo }\end{array}$ & $\stackrel{0}{\tilde{0}}$ & $\begin{array}{l}0 \\
\tilde{O} \\
z\end{array}$ & $\begin{array}{l}0 \\
\text { Lे } \\
z\end{array}$ & $\stackrel{0}{0}$ & 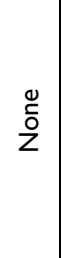 & $\begin{array}{l}0 \\
\stackrel{0}{0} \\
z\end{array}$ & $\begin{array}{l}\stackrel{0}{\tilde{\nu}} \\
\stackrel{0}{Z}\end{array}$ & $\begin{array}{l}\text { õ } \\
\text { ô }\end{array}$ & $\begin{array}{l}0 \\
\stackrel{0}{0} \\
z\end{array}$ & $\begin{array}{l}\stackrel{0}{0} \\
\stackrel{0}{z}\end{array}$ & $\begin{array}{l}\stackrel{0}{0} \\
\stackrel{0}{z}\end{array}$ & $\begin{array}{l}\stackrel{0}{0} \\
\text { Oे } \\
z\end{array}$ & $\begin{array}{l}\stackrel{0}{0} \\
\text { zo }\end{array}$ & $\begin{array}{l}\stackrel{0}{0} \\
\stackrel{0}{z}\end{array}$ & $\begin{array}{l}\stackrel{0}{0} \\
\text { zo }\end{array}$ \\
\hline 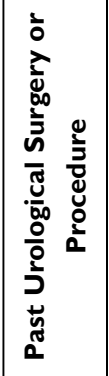 & 으 & io & $\stackrel{\circ}{z}$ & z & ㅇ & $\stackrel{\circ}{z}$ & $\stackrel{\circ}{z}$ & $\stackrel{\circ}{z}$ & $\stackrel{\circ}{z}$ & ㅇ & $\stackrel{\circ}{z}$ & $\stackrel{\circ}{z}$ & $\underbrace{\tilde{y}}$ & $\stackrel{\check{\nu}}{\check{\nu}}$ & 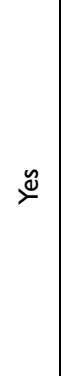 & $\stackrel{\circ}{z}$ & $\stackrel{\tilde{\nu}}{\nu}$ & $\stackrel{\circ}{z}$ & $\stackrel{\circ}{z}$ & ㅇ & ㅇ \\
\hline 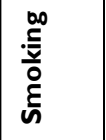 & $\begin{array}{l}\bar{\Phi} \\
\bar{\Xi}\end{array}$ & $\frac{\bar{d}}{\bar{\Xi}}$ & 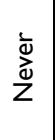 & 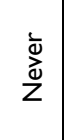 & 产 & 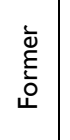 & 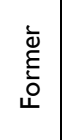 & $\frac{\bar{\Phi}}{\bar{\Xi}}$ & 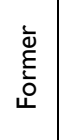 & 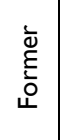 & $\frac{\bar{\Phi}}{\bar{\Xi}}$ & $\frac{\bar{y}}{\bar{\Xi}}$ & $\begin{array}{l}\overline{\bar{g}} \\
\bar{\Xi} \\
z\end{array}$ & 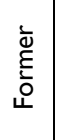 & $\frac{\bar{g}}{\stackrel{\bar{g}}{z}}$ & 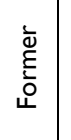 & $\begin{array}{l}\frac{\bar{d}}{\bar{d}} \\
z\end{array}$ & $\begin{array}{l}\stackrel{\bar{\nu}}{\bar{\nu}} \\
\bar{z}\end{array}$ & $\frac{\bar{d}}{\grave{U}_{Z}}$ & $\stackrel{\bar{\Phi}}{\stackrel{\bar{\Xi}}{Z}}$ & $\frac{\bar{d}}{\stackrel{\bar{U}}{Z}}$ \\
\hline $\begin{array}{ll}\overline{0} & \\
\bar{\delta} & 0 \\
\frac{\delta}{\alpha} & \supset\end{array}$ & 우 & 우 & $\stackrel{\stackrel{y}{*}}{\underbrace{2}}$ & $\stackrel{0}{z}$ & 우 & ㅇ & ㅇ & $\stackrel{\tilde{y}}{\nu}$ & 우 & $\underbrace{\mathscr{y}}_{\nu}$ & $\stackrel{\circ}{z}$ & $\stackrel{\circ}{z}$ & 으 & $\stackrel{0}{z}$ & $\stackrel{\circ}{z}$ & $\underbrace{y}_{\nu}$ & 우 & $\stackrel{\check{\nu}}{\nu}$ & ㅇ & zo & $\stackrel{\circ}{z}$ \\
\hline 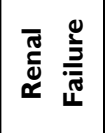 & ¿o & 우 & $\stackrel{\circ}{z}$ & $\stackrel{0}{z}$ & 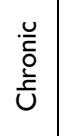 & $\begin{array}{l}\frac{U}{\bar{U}} \\
\frac{\partial}{\bar{U}} \\
\end{array}$ & $\stackrel{\circ}{z}$ & 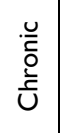 & ㅇ & 우 & $\stackrel{\circ}{z}$ & 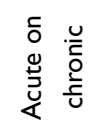 & $\stackrel{0}{z}$ & 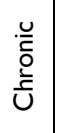 & $\stackrel{0}{z}$ & 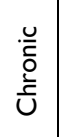 & 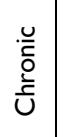 & 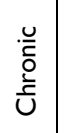 & 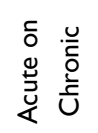 & $\stackrel{0}{z}$ & 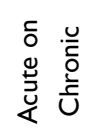 \\
\hline 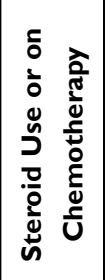 & ㅇ & zo & $\stackrel{\circ}{z}$ & z & ㅇ & z & ㅇ & ㅇ & ㅇ & zo & ㅇ & zo & 으 & $\stackrel{\check{\Perp}}{\check{\nu}}$ & ㅇ & $\stackrel{\varrho}{\check{\nu}}$ & zo & io & zo & zo & zo \\
\hline 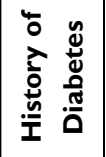 & ㅇ & $\stackrel{\check{\nu}}{\check{\nu}}$ & $\stackrel{\circ}{z}$ & $\stackrel{\tilde{y}}{\nu}$ & 우 & zo & ㅇ & ㅇ & ㅇ & zo & zo & $\stackrel{\circ}{z}$ & $\stackrel{\check{\nu}}{\check{\nu}}$ & z & ㅇ & $\stackrel{\mathscr{y}}{\nu}$ & zo & $\underbrace{\mathscr{\Xi}}_{\check{\nu}}$ & $\stackrel{\mathscr{y}}{\check{1}}$ & $\stackrel{\check{\nu}}{\nu}$ & z \\
\hline 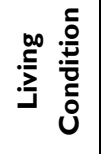 & 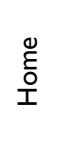 & $\begin{array}{l}0 \\
\stackrel{0}{\tilde{I}} \\
\text { I }\end{array}$ & $\begin{array}{l}\stackrel{0}{\tilde{E}} \\
\stackrel{0}{1}\end{array}$ & \begin{tabular}{|l|}
0 \\
$\stackrel{\Xi}{E}$ \\
Oे
\end{tabular} & I & 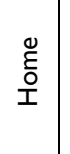 & $\frac{I}{Z}$ & $\begin{array}{l}0 \\
\stackrel{0}{\tilde{0}} \\
\text { I }\end{array}$ & $\begin{array}{l}\stackrel{0}{\tilde{E}} \\
\text { 움 }\end{array}$ & $\begin{array}{l}0 \\
\stackrel{0}{\tilde{E}} \\
\text { 임 }\end{array}$ & I & $\begin{array}{l}\stackrel{0}{\tilde{E}} \\
\text { 온 }\end{array}$ & $\begin{array}{l}\stackrel{0}{E} \\
\stackrel{0}{I}\end{array}$ & $\begin{array}{l}0 \\
\stackrel{0}{\tilde{E}} \\
\text { 이 }\end{array}$ & $\begin{array}{l}\stackrel{0}{\tilde{E}} \\
\text { 옴 }\end{array}$ & 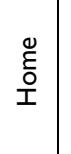 & 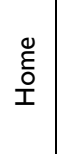 & $\begin{array}{l}\stackrel{0}{\tilde{E}} \\
\stackrel{0}{\underline{T}}\end{array}$ & $\begin{array}{l}\stackrel{0}{\tilde{E}} \\
\stackrel{0}{I}\end{array}$ & $\begin{array}{l}\stackrel{0}{\tilde{E}} \\
\stackrel{0}{I}\end{array}$ & $\begin{array}{l}0 \\
\stackrel{0}{\tilde{O}} \\
\text { I }\end{array}$ \\
\hline $\bar{\Sigma}$ & 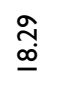 & 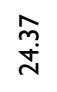 & $\bar{\sigma}$ & ָั & $\frac{m}{N}$ & นุํ & $\stackrel{\circ}{\circ}$ & $\stackrel{m}{\stackrel{m}{े}}$ & $\underset{\text { de }}{\stackrel{0}{~}}$ & $\stackrel{a}{\sim}$ & $\stackrel{\circ}{\dot{m}}$ & $\frac{R}{i}$ & $\stackrel{\stackrel{ \pm}{+}}{\sim}$ & 邖 & $\underset{\text { f̀ }}{+}$ & 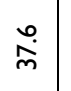 & $\stackrel{\tilde{n}}{\underline{\underline{n}}}$ & 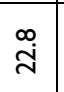 & $\frac{\bar{N}}{\dot{v}}$ & $\stackrel{\stackrel{\llcorner}{\infty}}{\wedge}$ & $\stackrel{\sim}{\sim}$ \\
\hline ڤ̊̀ & ч & $\Sigma$ & ч & ч & 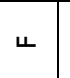 & 4 & ч & 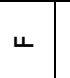 & 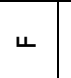 & 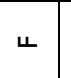 & $\Sigma$ & $\Sigma$ & $\Sigma$ & 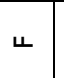 & $\Sigma$ & 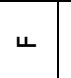 & 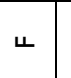 & 4 & ч & $\Sigma$ & $\Sigma$ \\
\hline$\stackrel{\infty}{⿺}$ & œ & $n$ & ถి & $\pi$ & 岕 & ঃ & tे & œ & $\stackrel{2}{*}$ & $\bar{\alpha}$ & $\tilde{\beta}$ & R & 。ํ & $\bar{\sigma}$ & $\hat{f}$ & $\stackrel{\star}{\star}$ & ঃ & $\stackrel{\infty}{\infty}$ & $\stackrel{\omega}{\infty}$ & Ф & $\varlimsup_{\infty}^{\infty}$ \\
\hline ن̃ & - & $N$ & $m$ & $\sigma$ & in & 0 & $n$ & $\infty$ & $\sigma$ & 으 & $=$ & $\simeq$ & $\underline{m}$ & $\dot{\Xi}$ & $\underline{\underline{n}}$ & $\underline{\bullet}$ & $\simeq$ & $\underline{\infty}$ & $\underline{\underline{a}}$ & 요 & $\bar{\sim}$ \\
\hline
\end{tabular}




\begin{tabular}{|c|c|c|c|c|c|c|c|c|c|c|c|c|c|c|c|c|}
\hline 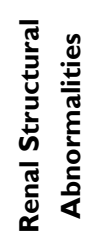 & $\begin{array}{l}\stackrel{0}{0} \\
\stackrel{0}{z}\end{array}$ & 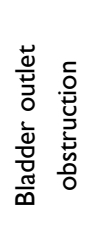 & 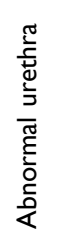 & $\begin{array}{l}\stackrel{0}{\check{0}} \\
\text { z }\end{array}$ & 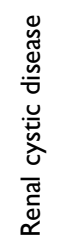 & 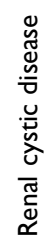 & 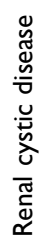 & $\begin{array}{l}0 \\
\stackrel{0}{0} \\
z\end{array}$ & $\begin{array}{l}\stackrel{0}{0} \\
\stackrel{0}{z}\end{array}$ & $\begin{array}{l}\stackrel{0}{0} \\
\stackrel{0}{Z}\end{array}$ & 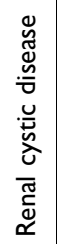 & $\begin{array}{l}0 \\
\stackrel{0}{0} \\
\text { Z }\end{array}$ & $\begin{array}{l}0 \\
\stackrel{0}{0} \\
\text { Zn }\end{array}$ & $\stackrel{0}{\stackrel{0}{0}}$ & $\stackrel{0}{0}$ & $\stackrel{0}{\tilde{O}}$ \\
\hline 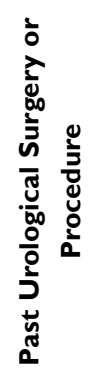 & $\stackrel{\Perp}{\nu}$ & ㅇ & zo & ż & z & 으 & 으 & 으 & ㅇ & 으 & 으 & zo & 을 & 은 & 으 & z \\
\hline 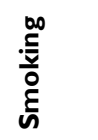 & $\begin{array}{l}\text { ऐ্য } \\
\text { ż }\end{array}$ & 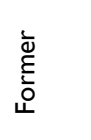 & 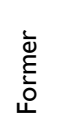 & 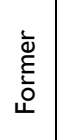 & 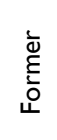 & 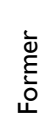 & 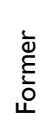 & 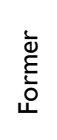 & 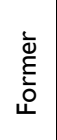 & $\begin{array}{l}\overline{\bar{y}} \\
\text { zù }\end{array}$ & $\begin{array}{l}\text { ऐ্े } \\
\text { żे }\end{array}$ & $\begin{array}{l}\grave{\bar{\nu}} \\
\overline{\mathrm{e}}\end{array}$ & 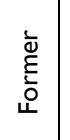 & 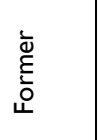 & 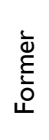 & 产 \\
\hline 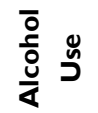 & zo & zo & zo & $\stackrel{\circ}{z}$ & zo & zo & zo & 을 & zo & zo & zo & zo & zo & 운 & zo & z \\
\hline 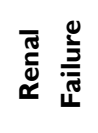 & 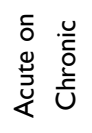 & zo & Z̊ & 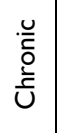 & $\frac{\breve{\Xi}}{\stackrel{\underline{\partial}}{\bar{U}}}$ & 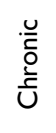 & zo & zo & zo & zo & 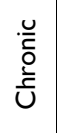 & 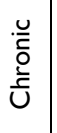 & $\begin{array}{l}\frac{U}{\bar{O}} \\
\frac{\partial}{U}\end{array}$ & 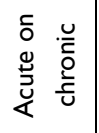 & zo & z \\
\hline
\end{tabular}

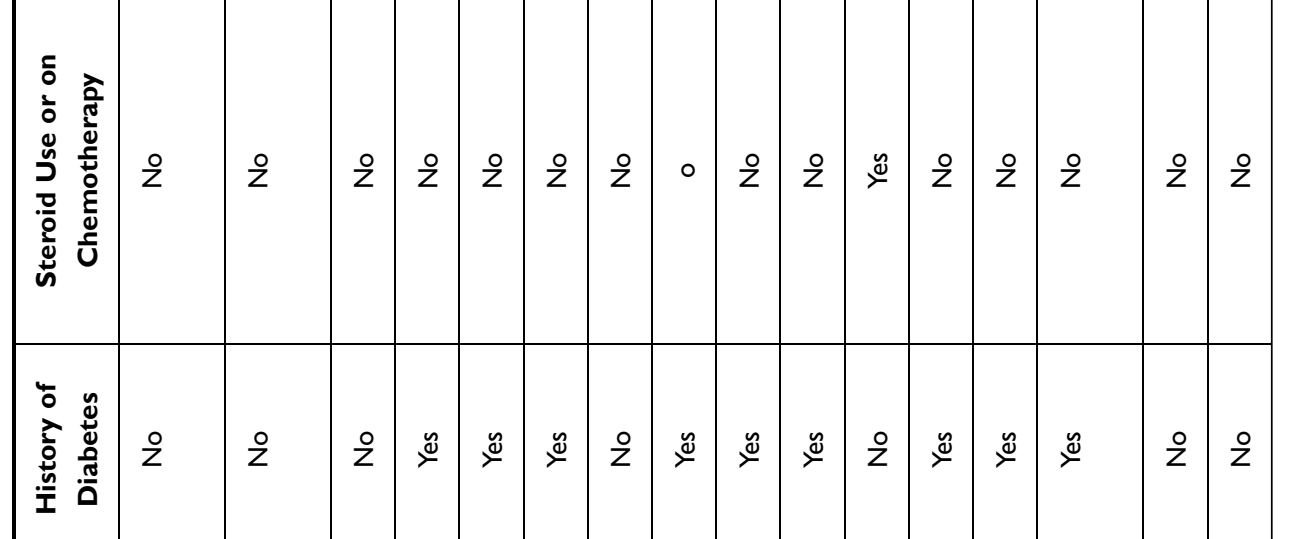

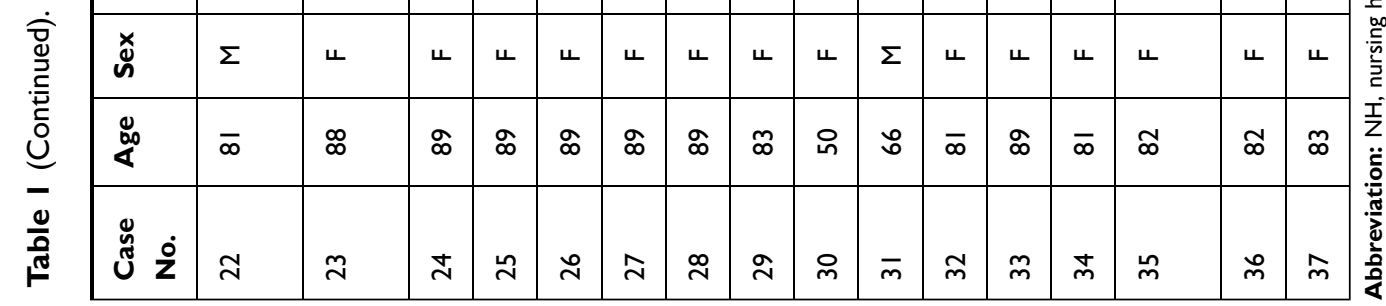




\section{Structural abnormalities of the genitourinary tract}

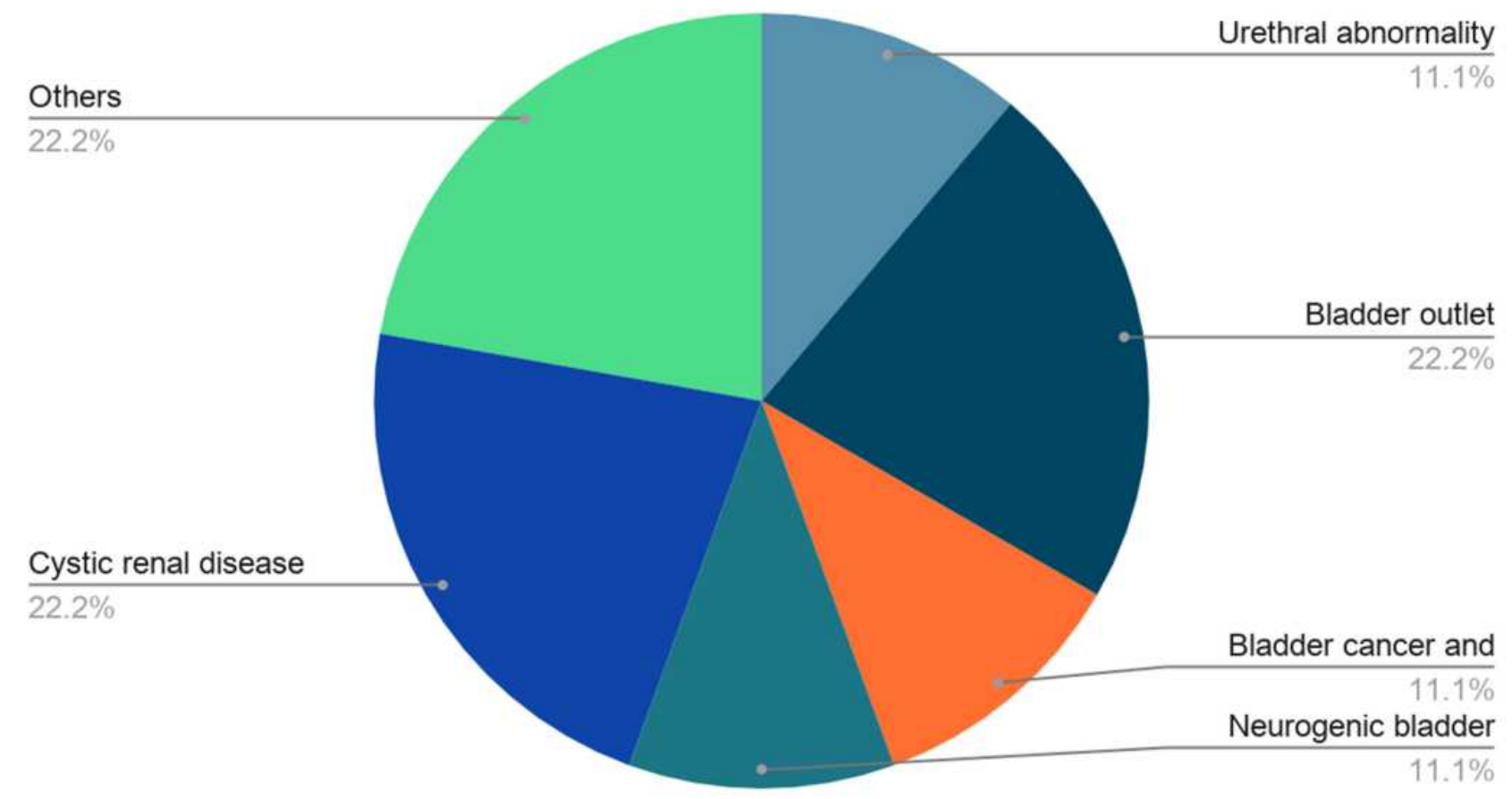

Figure I Underlying structural abnormality of the genitourinary tract.

\section{Clinical Features}

As shown in Table 2, most of the patients were asymptomatic at the time of presentation. The symptomatic patients presented with the following symptoms altered mental status (6 patients), generalized fatigue (5 patients), fever (4 patients), dysuria (2 patients), flank pain (1 patient), the combination of fever, suprapubic pain, and increased frequency (1 patient), flank pain and turbid urine (1 patient), and altered mental status and increased urinary frequency (1 patient).

Table 2 Clinical Features of Patients with R. planticola-Associated UTIs

\begin{tabular}{|l|l|l|}
\hline Symptom & $\begin{array}{l}\text { Number of Patients } \\
\text { (Total:37) }\end{array}$ & Percentage \\
\hline Asymptomatic & 16 & $43.24 \%$ \\
Bacteriuria & & $18.92 \%$ \\
Altered sensorium & 7 & $13.51 \%$ \\
Fatigue & 5 & $13.51 \%$ \\
Fever & 5 & $5.41 \%$ \\
Dysuria & 2 & $5.41 \%$ \\
Flank pain & 2 & $5.41 \%$ \\
Increased frequency & 2 & $5.41 \%$ \\
Suprapubic pain & 2 & $2.7 \%$ \\
Turbid urine & 1 & \\
\hline
\end{tabular}

Four patients were bradycardic, and 8 patients were tachycardic at the time of presentation. Only 8 patients were febrile $\left(>100.4^{\mathrm{O}} \mathrm{F}\right)$ at the time of presentation. Hypotension was present in 1 patient and the rest of the patients had systolic blood pressure greater than $90 \mathrm{~mm} \mathrm{Hg}$.

\section{Laboratory Data}

The laboratory results of the patients are depicted in Table 3. The mean white blood cell count was $8.96 \times 10^{9} / \mathrm{L}\left(2.3 \times 10^{9} /\right.$ $\left.\mathrm{L}-20.5 \times 10^{9} / \mathrm{L}\right)$, platelet count was $246 \times 10^{9} / \mathrm{L}\left(52 \times 10^{9} / \mathrm{L}\right.$ $\left.599 \times 10^{9} / \mathrm{L}\right)$, glucose was $152 \mathrm{mg} / \mathrm{dL}(49 \mathrm{mg} / \mathrm{dl}-684 \mathrm{mg} /$ $\mathrm{dL})$, creatinine was $1.31 \mathrm{mg} / \mathrm{dL}(0.53 \mathrm{mg} / \mathrm{dl}-6.3 \mathrm{mg} / \mathrm{dL})$, and GFR was $56.87 \mathrm{~mL} / \mathrm{min}(5.7 \mathrm{~mL} / \mathrm{min}-111.6 \mathrm{~mL} / \mathrm{min})$. A glycated hemoglobin level was measured only in 12 patients and the mean was $8.39 \%(5.3 \%-13.7 \%)$. An elevated lactate level was present in 3 patients.

\section{Culture, Coexisting Organism, and Antibiotic Sensitivity}

The microbiological data are detailed in Table 4. Resistance to ampicillin was found in 35 isolates, which seems to be an intrinsic character of the Raoultella spp., and 2 isolates were multidrug-resistant, but still susceptible to ciprofloxacin. Concomitant growth of other organisms was found in 9 urine 
Table 3 Laboratory Results

\begin{tabular}{|c|c|c|c|c|c|c|c|}
\hline $\begin{array}{l}\text { Case } \\
\text { No. }\end{array}$ & $\begin{array}{l}\text { WBC } \\
\text { (Cells/ } \\
\text { mL) }\end{array}$ & $\begin{array}{c}\text { Platelets } \\
\text { (Cells/ } \\
\mu L)\end{array}$ & $\begin{array}{l}\text { Hemoglobin } \\
\text { AIC } \\
(\%)\end{array}$ & $\begin{array}{c}\text { Blood Sugar at } \\
\text { Presentation }(\mathrm{mg} / \mathrm{dl})\end{array}$ & $\begin{array}{l}\text { Creatinine } \\
(\mathrm{mg} / \mathrm{dl})\end{array}$ & $\begin{array}{l}\text { Glomerular Filtrataion } \\
\text { Rate (mL/min) }\end{array}$ & $\begin{array}{c}\text { Lactic Acid } \\
\text { (mmol/L) }\end{array}$ \\
\hline I & 11.7 & 430 & Not done & 110 & 0.84 & 63.4 & 1.2 \\
\hline 2 & 14.4 & 231 & 6.5 & 171 & 0.87 & 85.6 & 1.4 \\
\hline 3 & 12.7 & 599 & Not done & 116 & 0.6 & 95.7 & 1.8 \\
\hline 4 & 7.7 & 277 & 8.5 & 213 & 1.07 & 52.6 & Not done \\
\hline 5 & 6.5 & 285 & Not done & 99 & 0.81 & 66.7 & Not done \\
\hline 6 & 10.2 & 199 & 5.9 & 96 & 1.38 & 33.6 & Not done \\
\hline 7 & 4.6 & 87 & Not done & 106 & 0.53 & 100.3 & Not done \\
\hline 8 & 8.2 & 269 & Not done & 49 & 1.16 & 43.5 & Not done \\
\hline 9 & 18.9 & 567 & Not done & 93 & 1.35 & 44.1 & Not done \\
\hline 10 & 12.7 & 253 & Not done & 119 & 1.73 & 29.6 & 1.8 \\
\hline II & 5.6 & 118 & 5.3 & 111 & 0.98 & 81.7 & 0.8 \\
\hline 12 & 12.8 & 330 & Not done & 126 & 1.63 & 42.1 & 1.5 \\
\hline 13 & 6.6 & 184 & 9 & 156 & 111.6 & 1 & 2.5 \\
\hline 14 & 10.6 & 259 & 7.2 & 95 & 1.55 & 36.1 & 2.1 \\
\hline 15 & 2.3 & 257 & Not done & 117 & 1.13 & 77 & Not done \\
\hline 16 & 8.3 & 400 & 8.8 & 216 & 1.32 & 39.6 & 1.2 \\
\hline 17 & 7 & 240 & Not done & 95 & 1.08 & 45.2 & Not done \\
\hline 18 & 9 & 52 & 6.2 & 235 & 1.23 & 46.3 & Not done \\
\hline 19 & 9.9 & 88 & Not done & 285 & 3.36 & 11.9 & Not done \\
\hline 20 & 7.3 & 270 & 13.7 & 684 & 0.81 & 92 & 1.8 \\
\hline 21 & 20.5 & 147 & Not done & 121 & 1.98 & 30.6 & 1.4 \\
\hline 22 & 19.5 & 199 & Not done & 123 & 1.57 & 40.7 & 2.3 \\
\hline 23 & 8 & 182 & Not done & 122 & 0.86 & 59.1 & Not done \\
\hline 24 & 6.4 & 183 & Not done & 115 & 0.84 & 60.3 & Not done \\
\hline 25 & 7.7 & 200 & 5.4 & $13 \mid$ & 1.32 & 35.7 & Not done \\
\hline 26 & 7.1 & 214 & Not done & 225 & 1.35 & 34.7 & 1.9 \\
\hline 27 & 8.7 & 201 & Not done & 123 & $\mathrm{I} .42$ & 38.5 & 1.5 \\
\hline 28 & 8.2 & 321 & Not done & 148 & 0.54 & 84.3 & 0.7 \\
\hline 29 & 5.8 & 109 & Not done & 176 & 0.63 & 83.5 & Not done \\
\hline 30 & 5.4 & 168 & Not done & 142 & 0.72 & 98.3 & Not done \\
\hline 31 & 6.2 & 225 & Not done & 129 & 1.01 & 77.1 & Not done \\
\hline 32 & 6.2 & 61 & Not done & 85 & 6.3 & 5.7 & 0.5 \\
\hline 33 & 3.3 & 75 & Not done & 124 & 1.75 & 25.4 & Not done \\
\hline 34 & 8.3 & 227 & II.I & 117 & 1.27 & 39.5 & Not done \\
\hline 35 & 12.1 & 243 & 13.2 & 251 & 1.83 & 25.2 & Not done \\
\hline 36 & 6.3 & 451 & Not done & 98 & 0.68 & 81.5 & Not done \\
\hline 37 & 5 & 503 & Not done & 104 & 0.59 & 85.4 & Not done \\
\hline
\end{tabular}

culture samples; namely, Escherichia coli (5 cultures), carbapenem-resistant Pseudomonas aeruginosa (1 culture), Enterococcus sp. (1 culture), Group B Streptococcus (1 culture), and mixed gram-positive organisms (1 culture). None of the patients had positive blood cultures.

\section{Clinical Course, Hospital Management,} and Outcome

The severity of illness varied widely ranging from asymptomatic bacteriuria to septic shock as depicted in Table 5. Antibiotics were administered to 24 patients 
Table 4 Microbiological Characteristics

\begin{tabular}{|c|c|c|c|c|}
\hline $\begin{array}{l}\text { Case } \\
\text { No. }\end{array}$ & Antibiotic Sensitivity Profile* & $\begin{array}{l}\text { Blood } \\
\text { Cultures }\end{array}$ & $\begin{array}{l}\text { Mode of } \\
\text { Urine } \\
\text { Collection }\end{array}$ & Other Organisms ${ }^{\#}$ \\
\hline I & Resistant to ampicillin & Negative & Clean catch & None \\
\hline 2 & Resistant to ampicillin & Not done & Clean catch & None \\
\hline 3 & Resistant to ampicillin & Not done & Clean catch & $<50,000$ Group B Streptococcus \\
\hline 4 & Resistant to ampicillin & Not done & Clean catch & None \\
\hline 5 & $\begin{array}{l}\text { Resistant to ampicillin, cefazolin, ceftazidime, gentamicin. } \\
\text { Intermediate sensitivity to tobramycin. }\end{array}$ & Not done & Clean catch & Escherichia coli \\
\hline 6 & Resistant to ampicillin. & Not done & Clean catch & None \\
\hline 7 & Resistant to ampicillin & Negative & Clean catch & Escherichia coli \\
\hline 8 & Resistant to ampicillin & Not done & Clean catch & $\begin{array}{l}\text { Escherichia coli, } 50,000-100,000 \\
\text { CFU mixed gram positive } \\
\text { orgnisms }\end{array}$ \\
\hline 9 & Resistant to ampicillin & Not done & Clean catch & None \\
\hline 10 & Resistant to ampicillin & Not done & Clean catch & None \\
\hline II & Resistant to ampicillin & Not done & Clean catch & None \\
\hline 12 & Resistant to ampicillin & Not done & $\begin{array}{l}\text { Nephrostomy } \\
\text { tube }\end{array}$ & Enterococcus fecalis \\
\hline 13 & $\begin{array}{l}\text { Resistant to ampicillin, cefazolin, ceftazidime, cefoxitin, gentamicin, } \\
\text { trimethoprim-sulfamethoxazole, aztreonam, meropenem, piperacillin- } \\
\text { tazobactam. Intermediate sensitivity to tobramycin; }\end{array}$ & Negative & Foley catheter & CRE Pseudomonas aeurogenosa. \\
\hline 14 & Resistant to ampicillin & Negative & Clean catch & None \\
\hline 15 & Resistant to ampicillin & Negative & Clean catch & Escherichia coli \\
\hline 16 & Resistant to ampicillin & Not done & Foley catheter & Escherichia coli \\
\hline 17 & Resistant to ampicillin & Not done & Clean catch & None \\
\hline 18 & Resistant to ampicillin & Not done & $\begin{array}{l}\text { Straight } \\
\text { catheterization }\end{array}$ & None \\
\hline 19 & Resistant to ampicillin & Not done & Clean catch & None \\
\hline 20 & Resistant to ampicillin & Negative & Clean catch & None \\
\hline 21 & Resistant to ampicillin & Negative & $\begin{array}{l}\text { Straight } \\
\text { catheterization }\end{array}$ & None \\
\hline 22 & Resistant to ampicillin & Negative & Clean catch & $\begin{array}{l}50,000-100,000 \mathrm{CFU} \\
\text { Mixed gram positive and gram } \\
\text { negative. }\end{array}$ \\
\hline 23 & Resistant to ampicillin & Not done & Clean catch & None \\
\hline 24 & Resistant to ampicillin & Not done & Clean catch & None \\
\hline 25 & Resistant to ampicillin & Negative & Clean catch & None \\
\hline
\end{tabular}


Table 4 (Continued).

\begin{tabular}{|l|l|l|l|l|}
\hline $\begin{array}{l}\text { Case } \\
\text { No. }\end{array}$ & Antibiotic Sensitivity Profile* & $\begin{array}{l}\text { Blood } \\
\text { Cultures }\end{array}$ & $\begin{array}{l}\text { Mode of } \\
\text { Urine } \\
\text { Collection }\end{array}$ & Other Organisms \\
\hline 26 & Resistant to ampicillin & Not done & Clean catch & None \\
\hline 27 & Resistant to ampicillin & Not done & Foley catheter & None \\
\hline 28 & Resistant to ampicillin & Not done & Clean catch & None \\
\hline 29 & Resistant to ampicillin & Not done & $\begin{array}{l}\text { Straight } \\
\text { catheterization }\end{array}$ & None \\
\hline 30 & Resistant to ampicillin & Not done & Clean catch & None \\
\hline 31 & Resistant to ampicillin & Not done & Foley catheter & None \\
\hline 32 & Resistant to ampicillin & Negative & Clean catch & None \\
\hline 33 & Resistant to ampicillin & Negative & Clean catch & None \\
\hline 34 & Resistant to ampicillin & Not done & Clean catch & None \\
\hline 35 & Resistant to ampicillin & Negative & Foley catheter & None \\
\hline 36 & Resistant to ampicillin & Not done & Clean catch & None \\
\hline 37 & Resistant to ampicillin & Not done & Clean catch & None \\
\hline
\end{tabular}

Notes: *The antibiotics for which the sensitivities were analyzed were ampicillin, cefoxitin, cefazolin, ceftazidime, gentamicin, ciprofloxacin, nitrofurantoin, trimethoprimsulfamethoxazole (Bactrim), aztreonam, meropenem, piperacillin-tazobactam. Due to lack of space, only the resistance pattern has been documented in the table It is implied that the isolate was sensitive to other antibiotics mentioned above. ${ }^{\#}$ All the organisms in the urine culture were greater than $10^{5}$ colony forming units unless specified. Abbreviation: CFU, colony forming units.

while 13 patients did not receive any antibiotics. The mean duration of antibiotic administration was 7.54 days. Ciprofloxacin was the most commonly used antibiotic and was administered to 9 patients. Ceftazidime/ avibactam was used in the UTI caused by drugresistant isolates. All patients responded to treatment. The mean length of stay was 3.18 days.

\section{Discussion}

Urinary tract infections can range from simple asymptomatic bacteriuria to complicated ascending tract infections leading to bacteremia and sepsis. ${ }^{18}$ The risk of complications is more pronounced in diabetics, elderly people with indwelling catheters, and immunocompromised individuals. ${ }^{19-21}$ Raoultella planticola has been rarely reported to be significantly associated with UTI. To the best of our knowledge, this is the first review of the clinical characteristics of $R$. planticola-associated UTIs.

$R$. planticola UTI seems to occur approximately three times more common in females in our study population, which mirrors the gender distribution for UTIs with other organisms. ${ }^{22}$
Advanced age, immunocompromised condition like cancer, diabetes mellitus and impaired renal function were noted to be significant risk factors for developing $R$. planticola UTI, similar to the risk factors in UTIs due to other organisms. ${ }^{23,42}$ The mean age of our study population was 77 years. A diagnosis of diabetes mellitus was present in $43.24 \%(16 / 37)$ of the patients and $21 \%(8 / 37)$ had a glycated hemoglobin $(\mathrm{HbA} 1 \mathrm{C})>6 \%$.

A BMI of greater than $25 \mathrm{~kg} / \mathrm{m}^{2}$ was present in $51.35 \%$ (19/37) of patients and the mean BMI of this cohort was $26.64 \mathrm{~kg} / \mathrm{m}^{2}$. Our study was underpowered to determine if obesity was an independent risk factor.

A large study of UTI in diabetics, done in Germany, has suggested that a GFR of less than $60 \mathrm{~mL} / \mathrm{min}$ increases the risk of UTI. ${ }^{23}$ However, it is unclear if chronic renal insufficiency is a risk independent of age and diabetes mellitus. ${ }^{24}$ Twenty-three patients (including one patient who was post-renal transplant) in our study population had chronic kidney disease (GFR $<60 \mathrm{~mL} / \mathrm{min}$ ). In the subset of patients with chronic renal insufficiency, 52\% (12/23) did not have a diagnosis of diabetes mellitus. Therefore, it is likely that impaired renal function 
Table 5 The Clinical Course of the Patients with R. planticola-Associated UTIs

\begin{tabular}{|c|c|c|c|c|c|}
\hline $\begin{array}{l}\text { Case } \\
\text { No. }\end{array}$ & Severity of IIIness & Antibiotic Administered & $\begin{array}{l}\text { Length of } \\
\text { Stay }\end{array}$ & $\begin{array}{l}\text { Duration of } \\
\text { Antibiotics }\end{array}$ & Outcome \\
\hline 1 & Acute cystitis & Ciprofloxacin & 2 & 7 & Discharged home \\
\hline 2 & Acute cystitis & Ciprofloxacin & 3 & 7 & Discharged home \\
\hline 3 & $\begin{array}{l}\text { Asymptomatic } \\
\text { bacteriuria }\end{array}$ & None & 4 & - & Discharged home \\
\hline 4 & $\begin{array}{l}\text { Asymptomatic } \\
\text { bacteriuria }\end{array}$ & None & 2 & - & Discharged home \\
\hline 5 & $\begin{array}{l}\text { Asymptomatic } \\
\text { bacteriuria }\end{array}$ & None & 5 & - & Discharged back to $\mathrm{NH}$. \\
\hline 6 & Acute cystitis & Ciprofloxacin & 3 & 5 & Discharged home \\
\hline 7 & Acute cystitis & Ciprofloxacin & 5 & 7 & Discharged back to $\mathrm{NH}$. \\
\hline 8 & Acute cystitis & Ciprofloxacin & 5 & 5 & Discharged home \\
\hline 9 & $\begin{array}{l}\text { Asymptomatic } \\
\text { bacteriuria }\end{array}$ & Nitrofurantoin & 6 & 10 & Discharged home \\
\hline 10 & Acute cystitis & Levofloxacin, Cefdinir & 4 & I & Discharged home \\
\hline II & Acute cystitis & Cephalexin & 0 & & $\begin{array}{l}\text { Left against medical advice from } \\
\text { the ED. }\end{array}$ \\
\hline 12 & $\begin{array}{l}\text { Asymptomatic } \\
\text { bacteriuria }\end{array}$ & Ampicillin & 7 & 3 & Discharged home \\
\hline 13 & Septic shock & Ceftazidime/Avibactam & 8 & 14 & Discharged home \\
\hline 14 & $\begin{array}{l}\text { Asymptomatic } \\
\text { bacteriuria }\end{array}$ & Ampicillin + ceftazidime & 4 & 3 & Discharged home \\
\hline 15 & $\begin{array}{l}\text { Asymptomatic } \\
\text { bacteriuria }\end{array}$ & None & & & Discharged home \\
\hline 16 & Acute cystitis & Ciprofloxacin & 4 & 10 & Discharged home. \\
\hline 17 & Acute cystitis & None & 0 & & $\begin{array}{l}\text { Left against medical advice from } \\
\text { the ED. }\end{array}$ \\
\hline 18 & $\begin{array}{l}\text { Asymptomatic } \\
\text { bacteriuria }\end{array}$ & None & 0 & & Discharged home from the ED \\
\hline 19 & $\begin{array}{l}\text { Asymptomatic } \\
\text { bacteriuria }\end{array}$ & None & 4 & 0 & Discharged home \\
\hline 20 & Sepsis & Ceftriaxone & 3 & 7 & Discharged home \\
\hline 21 & Sepsis & Ceftazidime & 6 & 14 & Discharged home \\
\hline 22 & Acute cystitis & $\begin{array}{c}\text { Ceftazidime for } 3 \text { days then } \\
\text { ciprofloxacin. }\end{array}$ & 3 & 10 & Discharged to short term rehab \\
\hline 23 & $\begin{array}{l}\text { Asymptomatic } \\
\text { bacteriuria }\end{array}$ & None & 2 & 0 & Discharged home \\
\hline
\end{tabular}

(Continued) 
Table 5 (Continued).

\begin{tabular}{|c|c|c|c|c|c|}
\hline $\begin{array}{l}\text { Case } \\
\text { No. }\end{array}$ & Severity of Illness & Antibiotic Administered & $\begin{array}{l}\text { Length of } \\
\text { Stay }\end{array}$ & $\begin{array}{l}\text { Duration of } \\
\text { Antibiotics }\end{array}$ & Outcome \\
\hline 24 & $\begin{array}{l}\text { Asymptomatic } \\
\text { bacteriuria }\end{array}$ & None & $0(\mathrm{ED})$ & 0 & Discharged home \\
\hline 25 & Acute cystitis & $\begin{array}{c}\text { Ceftazidime for } 3 \text { days then } \\
\text { cefpodoxime }\end{array}$ & 4 & 7 & Discharged back to $\mathrm{NH}$ \\
\hline 26 & Acute cystitis & Nitrofurantoin & 0 (ED) & 7 & Discharged back to $\mathrm{NH}$ \\
\hline 27 & Acute cystitis & $\begin{array}{c}\text { Ceftazidime for } 3 \text { days then } \\
\text { cefpodoxime }\end{array}$ & 3 & 10 & Discharged back to $\mathrm{NH}$ \\
\hline 28 & $\begin{array}{l}\text { Asymptomatic } \\
\text { bacteriuria }\end{array}$ & Ciprofloxacin & 4 & 5 & Discharged back to $\mathrm{NH}$ \\
\hline 29 & Acute cystitis & $\begin{array}{l}\text { Ceftazidime for } 4 \text { days then } \\
\text { cefpodoxime }\end{array}$ & 5 & 10 & Discharged to short term rehab. \\
\hline 30 & Acute cystitis & Ciprofloxacin & 0 (ED) & 7 & Discharged home \\
\hline 31 & $\begin{array}{c}\text { Asymptomatic } \\
\text { bacteriuria }\end{array}$ & None & 2 & 0 & Discharged to short term rehab. \\
\hline 32 & Septic shock & Ceftazidime & 4 & 7 & Discharged back to $\mathrm{NH}$. \\
\hline 33 & $\begin{array}{l}\text { Asymptomatic } \\
\text { bacteriuria }\end{array}$ & None & 4 & 0 & Discharged home. \\
\hline 34 & $\begin{array}{l}\text { Asymptomatic } \\
\text { bacteriuria }\end{array}$ & None & 3 & 0 & Discharged home. \\
\hline 35 & Acute cystitis & $\begin{array}{c}\text { Ceftazidime for } 3 \text { days then } \\
\text { cefpodoxime. }\end{array}$ & 3 & 7 & Discharged home. \\
\hline 36 & $\begin{array}{l}\text { Asymptomatic } \\
\text { bacteriuria }\end{array}$ & None & 2 & 0 & Discharged home. \\
\hline 37 & Acute cystitis & Cefpodoxime & 4 & 7 & Discharged home. \\
\hline
\end{tabular}

Abbreviations: $\mathrm{NH}$, nursing home; $\mathrm{ED}$, emergency department.

increases the risk of $R$. planticola-associated UTIs. However, our study was not powered to make that determination.

In our study, the most common presentation was asymptomatic bacteriuria (16/37, 43.24\%). However, 4 patients $(10.81 \%)$ in the study population fulfilled the SIRS criteria for sepsis. Of those, 2 patients (5.4\%) went into septic shock requiring aggressive intravenous fluid therapy and/or pressor support. Levy et al found that approximately $9-31 \%$ of all cases of sepsis can be attributed to urinary tract infection (depending on the geographical region). ${ }^{25}$ Based on studies, Klebsiella spp. is responsible for $15 \%$ of all cases of urosepsis. ${ }^{26,27}$ Due to its close similarity to Klebsiella spp., it is possible that many of those could have been due to R. planticola and may have been misdiagnosed in the past. There have been a few case reports of Raoultella bacteremia. ${ }^{6-9,28,29}$ No cases of bacteremia secondary to UTI were observed in our study. With the increasing use of techniques such as Matrix-Assisted Desorption Ionization-Time of Flight Mass Spectrometry (MALDITOF MS), $R$. planticola is being detected more frequently than in the past. ${ }^{28,29}$

Multiple studies have demonstrated that UTIs present differently in the elderly. ${ }^{30,31}$ A study performed by D'Agata et al in-nursing home residents showed that altered mental status is by far the commonest presentation (approximately 40\%). The classical UTI symptoms of 
dysuria $(3.8 \%)$, costovertebral tenderness $(2.3 \%)$, urinary frequency $(1.5 \%)$, urgency $(0 \%)$ and suprapubic pain $(0 \%)$ are much less common in adults greater than 65 years. ${ }^{30}$ Our study demonstrated similar findings.

As mentioned above, due to phylogenetic similarities, it is difficult to differentiate between Klebsiella spp. and Raoultella spp. by microscopy. The biochemical tests to differentiate Raoultella spp. from Klebsiella spp. such as ornithine decarboxylase activity, histamine or D-melezitose utilization, is not routinely available in commercial test kits. ${ }^{26,27}$ MALDI-TOF MS has emerged, in recent years, as a faster technique to identify Raoultella spp. and its reliability has been proven by multiple studies. $^{5,32-34}$ It essentially involves taking a sample from a bacterial colony, absorbing the lysed bacterial proteins onto a matrix, followed by ionizing and desorbing it with a laser. The resultant plume is analyzed through mass spectrometry to detect the signature pattern that identifies the bacteria. An additional advantage is the rapid detection of resistant strains. This technique is very effective in gram-negative bacteria, even with microcolonies. ${ }^{35}$ One limitation is the need for pure colonies; mixed colonies can lead to erroneous results.

In our laboratory, we used the VITEK $^{\circledR} 2$ compact automated system microbial identification (ID) and antibiotic susceptibility testing (AST) card for identification of organisms. The VITEK $^{\circledR} 2$ compact automated system uses 47 biochemical tests on a compact card specifically designed to identify gram-negative bacilli and their sensitivity. This technique usually identifies the organisms within 10 hours. $^{36} \mathrm{~A}$ frequently encountered problem with the system is the misidentification of Raoultella spp. as Klebsiella spp. ${ }^{32}$ To avoid such errors, Analytical Profile Index-20E (API-20E) was used to differentiate between members of the Enterobacteriaceae family in our laboratory. This technique uses a miniaturized version of 20 biochemical tests. The positive and negative results are compiled to obtain a unique profile code used to identify the organism. The sensitivity for identification of Raoultella spp. is $93.3 \%$ in VITEK ${ }^{\circledR} 2$ compact automated system, and $97.4 \%$ in the MALDI-TOF MS method. Though MALDI-TOF is better than VITEK ${ }^{\circledR} 2$ compact automated system in terms of sensitivity, both systems detect Raoultella spp. with a high degree of accuracy and the high initial cost of the MALDI-TOF system has restricted its use mostly to higher centres.

R. planticola is intrinsically resistant to ampicillin due to the over-expression of chromosomally encoded class-A $\beta$-lactamase. ${ }^{31,32}$ The other two major groups of resistance genes seen in Raoultella spp. are extended-spectrum $\beta$ lactamase (ESBL) and carbapenemase genes. Due to the ubiquitous nature of Raoultella spp, there is a very high risk of them acting as environmental reservoirs for resistance genes, such as carbapenemase. ${ }^{37,38}$ An analysis of sewage water from a tertiary centre in Spain revealed the presence of multiple carbapenemases producing Raoultella spp. ${ }^{39}$ These resistance genes can then be carried on mobile genetic elements like transposons and plasmids which are capable of transforming naive bacteria. In our study population, 2 patients had multidrug-resistant R. planticola-associated UTI. Both of these strains were resistant to ceftazidime which is routinely used in our institution to cover urinary gram-negative bacteria as per our hospital antibiogram. Though not reported, we can assume that this isolate probably had ESBL resistance gene/genes. Out of these two patients, one had a hospitalacquired catheter-associated UTI with a coexisting carbapenem-resistant Pseudomonas aeruginosa. The other patient had a coexisting Escherichia coli infection.

In addition to this, another 9 patients had a concomitant growth of a second organism found on urine culture. This is particularly concerning as mobile genetic elements, especially plasmids (chiefly IncF, IncI, IncA/C, IncL, IncN, and IncH plasmids) can transmit resistance genes between Enterobacteriaceae. ${ }^{40,41}$

\section{Limitations of the Study}

Due to the low prevalence of $R$. planticola-associated UTIs in general, the sample size was small in our study. We were not able to establish the statistical significance of our findings as a result of the small sample size. As this was a retrospective study, we encountered missing data. For example, not all patients had glycated hemoglobin levels analyzed during their hospitalization. Information on whether a urinary catheter was placed during the current hospitalization or whether it was chronic was not documented in many patient's charts. This left us unable to determine whether some of the UTIs were true infections or colonization that was discovered incidentally. Similarly, details on the structural abnormalities of the genitourinary tract and outpatient urological procedures were missing sometimes. The samples were analyzed by VITEK ${ }^{\circledR} 2$ compact automated system microbial identification (ID) and antibiotic susceptibility testing (AST) card which is less accurate than a MALDI-TOF MS. 


\section{Conclusion}

Our study revealed that patients with $R$. planticola UTI had higher proportion of diabetes mellitus, renal failure compared to the general population. Unlike prior case reports, most of the infections occurred in immunocompetent patients. Our study also confirms the intrinsic resistance to ampicillin of $R$. species, which has been documented previously in the literature. Surprisingly, Escherichia coli seems to coexist with Raoultella spp. in a significant number of cultures. A worrying finding was the presence of multidrug-resistant isolates, one of which was associated with multidrug-resistant Pseudomonas aeruginosa, which raises concern for the transmission of resistance genes. This raises concern for the transmission of resistance genes. As our study was limited by relatively small sample size and the retrospective nature of the analysis, larger studies would help us further define the observations noted in this study.

\section{Ethical Statement}

The article doesn't contain the participation of any human being and animal. As per our IRB, we did not require to take patient consent for the retrospective study for the electronic chart review. The study team ensured to maintain the patient data confidentiality and also was compliant with the Declaration of Helsinki.

\section{Institutional Approval}

IRB approval taken for this project from our hospital research approval team (MetroWest Medical Centre Institutional Review Board, IRB \#2020 -142).

\section{Author Contributions}

All authors made substantial contributions to conception and design, acquisition of data, or analysis and interpretation of data; took part in drafting the article or revising it critically for important intellectual content; agreed to submit to the current journal; gave final approval of the version to be published; and agreed to be accountable for all aspects of the work.

\section{Funding}

No funding used.

\section{Disclosure}

Authors have no conflicts of interest to declare.

\section{References}

1. Sahu KK, Sherif AA, Davaro R. A rare cause of cellulitis: photobacterium damselae. $J$ Microsc Ultrastruct. 2020;8(1):25-26. doi:10.4103/JMAU.JMAU_63_18

2. Sahu KK, Mishra AK, Lal A, Abraham GM. Mycobacterium avium complex: a rare cause of pancytopenia in HIV Infection. $J$ Microsc Ultrastruct. 2020;8(1):27-30.

3. Howell C, Fakhoury J. A case of Raoultella planticola causing a urinary tract infection in a pediatric patient. Transl Pediatr. 2017;6(2):102-103.

4. Castillo-Macías A, Flores-Aréchiga A, Llaca-Díaz J, Pérez-Chávez F, Casillas-Vega N. Microbiology of genus Raoultella, clinical features and difficulties in its diagnosis. Rev Medica Inst Mex Seguro Soc. 2018;56(5):486-490.

5. Sękowska A. Raoultella spp.-clinical significance, infections and susceptibility to antibiotics. Folia Microbiol (Praha). 2017;62 (3):221-227.

6. Hong G, Yong HJ, Lee D, et al. Clinical characteristics and treatment outcomes of patients with pneumonia caused by Raoultella planticola. J Thorac Dis. 2020;12(4):1305-1311.

7. Ulukent SC, Sarici İS, Alper Sahbaz N, Ozgun YM, Akca O, Sanlı K. Is it necessary to specifically define the cause of surgically treated biliary tract infections? A rare case of raoultella planticola cholecystitis and literature review. Case Rep Infect Dis. 2017;2017:4181582.

8. Chun S, Yun JW, Huh HJ, Lee NY. Low virulence? Clinical characteristics of Raoultella planticola bacteremia. Infection. 2014;42 (5):899-904.

9. AlSweed A, Alghamdi A, Tufenkeji H, Al-Hajjar S. The first case of Raoultella planticola infective endocarditis in a 4 year old child: a case report and review of literature. Int J Pediatr Adolesc Med. 2018;5(1):28-30.

10. Mehmood H, Pervin N, Israr Ul Haq M, Kamal KR, Marwat A, Khan M. A rare case of raoultella planticola urinary tract infection in a patient with immunoglobulin A nephropathy. $J$ Investig Med High Impact Case Rep. 2018;6:2324709618780422.

11. Harmon SL, Nadeem I. Recurrent urinary tract infections caused by Raoultella planticola after kidney transplant. Transpl Infect Dis off $J$ Transplant Soc. 2019;21(6):e13196.

12. Fager C, Yurteri-Kaplan L. Urinary tract infection with rare pathogen Raoultella Planticola: a post-operative case and review. Urol Case Rep. 2019;22:76-79.

13. Skelton WP, Taylor Z, Hsu J. A rare case of Raoultella planticola urinary tract infection in an immunocompromised patient with multiple myeloma. IDCases. 2017;8:9-11.

14. Gangcuangco LMA, Saul ZK. A novel case of Raoultella planticola urinary tract infection in a female: comment on "Nosocomial pneumonia caused by carbapenem-resistant Raoultella planticola: a case report and literature review.". Infection. 2015;43(5):621-622.

15. Tuğcu M, Ruhi C, Gokce AM, Kara M, Aksaray S. A case of urinary tract infection caused by Raoultella planticola after a urodynamic study. Braz J Infect Dis off Publ Braz Soc Infect Dis. 2017;21 (2):196-198.

16. Olson DS, Asare K, Lyons M, Hofinger DM. A novel case of Raoultella planticola urinary tract infection. Infection. 2013;41 (1):259-261.

17. Demiray T, Koroglu M, Ozbek A, Altindis M. A rare cause of infection, Raoultella planticola: emerging threat and new reservoir for carbapenem resistance. Infection. 2016;44(6):713-717.

18. Sahu KK, Mishra AK, Lal A. Clinical significance of aerococcus-related infections: an emerging threat. $J$ Pediatr Infect Dis Soc. 2019;8(6):578.

19. Sahu KK, Prakash G, Khadwal A, Varma SC, Malhotra P, Rare A. Case of hemorrhagic cystitis in allogeneic hematopoietic stem cell transplant patient. Indian J Hematol Blood Transfus off J Indian Soc Hematol Blood Transfus. 2016;32(Suppl 1):196-200. 
20. Sahu KK, Tsitsilianos N, Moselle L, Mishra AK. Septic arthritis of hip joint and its devastating complications. BMJ Case Rep. 2020;13 (2).

21. Sharma S, Singh P, Sahu KK, Rajwanshi A, Malhotra P, Naseem S. Histoplasmosis in pleural effusion in a 23-year-old man with mixed-phenotype acute leukemia. Lab Med. 2017;48(3):249-252.

22. Magliano E, Grazioli V, Deflorio L, et al. Gender and age-dependent etiology of community-acquired urinary tract infections. ScientificWorldJournal. 2012;2012:349597.

23. Wilke T, Boettger B, Berg B, et al. Epidemiology of urinary tract infections in type 2 diabetes mellitus patients: an analysis based on a large sample of 456,586 German T2DM patients. $J$ Diabetes Complications. 2015;29(8):1015-1023.

24. Gilbert DN. Urinary tract infections in patients with chronic renal insufficiency. Clin J Am Soc Nephrol CJASN. 2006;1(2):327-331.

25. Levy MM, Artigas A, Phillips GS, et al. Outcomes of the Surviving Sepsis Campaign in intensive care units in the USA and Europe: a prospective cohort study. Lancet Infect Dis. 2012;Dec;12 (12):919-924.

26. Rosenthal EJK. [Epidemiology of septicaemia pathogens]. Dtsch Med Wochenschr 1946. 2002;127(46):2435-2440. German.

27. Wagenlehner FME, Lichtenstern C, Rolfes C, et al. Diagnosis and management for urosepsis. Int J Urol off J Jpn Urol Assoc. 2013;20 (10):963-970.

28. Singhal N, Kumar M, Kanaujia PK, Virdi JS. MALDI-TOF mass spectrometry: an emerging technology for microbial identification and diagnosis. Front Microbiol. 2015;6:791.

29. Ponce-Alonso M, Rodríguez-Rojas L, Del Campo R, Cantón R, Morosini M-I. Comparison of different methods for identification of species of the genus Raoultella: report of 11 cases of Raoultella causing bacteraemia and literature review. Clin Microbiol Infect. 2016;22(3):252-257. doi:10.1016/j.cmi.2015.10.035

30. D’Agata E, Loeb MB, Mitchell SL. Challenges in assessing nursing home residents with advanced dementia for suspected urinary tract infections. J Am Geriatr Soc. 2013;61(1):62-66.

31. Rowe TA, Juthani-Mehta M. Diagnosis and management of urinary tract infection in older adults. Infect Dis Clin North Am. 2014;28 (1):75-89.

32. Sekowska A, Mikucka A, Gospodarek-Komkowska E. Identification of Raoultella spp.: comparison of three methods. Indian $\mathrm{J} \mathrm{Med}$ Microbiol. 2018;36(2):197-200.
33. Hansen DS, Aucken HM, Abiola T, Podschun R. Recommended test panel for differentiation of Klebsiella species on the basis of a trilateral interlaboratory evaluation of 18 biochemical tests. J Clin Microbiol. 2004;42(8):3665-3669.

34. Richter SS, Sercia L, Branda JA, et al. Identification of Enterobacteriaceae by matrix-assisted laser desorption/ionization time-of-flight mass spectrometry using the VITEK MS system. Eur J Clin Microbiol Infect Dis off Publ Eur Soc Clin Microbiol. 2013;32 (12):1571-1578.

35. Faron ML, Buchan BW, Ledeboer NA. Matrix-assisted laser desorption ionization-time of flight mass spectrometry for use with positive blood cultures: methodology, performance, and optimization. J Clin Microbiol. 2017;55(12):3328-3338.

36. Funke G, Funke-Kissling P. Evaluation of the new VITEK 2 card for identification of clinically relevant gram-negative rods. $J$ Clin Microbiol. 2004;42(9):4067-4071.

37. Tufa TB, Fuchs A, Feldt T, et al. CTX-M-9 group ESBL-producing Raoultella planticola nosocomial infection: first report from sub-Saharan Africa. Ann Clin Microbiol Antimicrob. 2020;19(1):36.

38. Walckenaer E, Poirel L, Leflon-Guibout V, Nordmann P, NicolasChanoine M-H. Genetic and biochemical characterization of the chromosomal class A beta-lactamases of Raoultella (formerly Klebsiella) planticola and Raoultella ornithinolytica. Antimicrob Agents Chemother. 2004;48(1):305-312.

39. Yao Y, Lazaro-Perona F, Falgenhauer L, et al. Insights into a Novel blaKPC-2-Encoding IncP-6 plasmid reveal carbapenem-resistance circulation in several Enterobacteriaceae species from wastewater and a Hospital Source in Spain. Front Microbiol. 2017;8:1143.

40. Carattoli A, Villa L, Fortini D, García-Fernández A. plasmids involved in the transmission and spread of antimicrobial resistance in Enterobacteriaceae. Plasmid. 2018.

41. Iredell J, Brown J, Tagg K. Antibiotic resistance in Enterobacteriaceae: mechanisms and clinical implications. $B M J$. 2016;8(352):h6420.

42. Jandial A, Mishra K, Sandal R, Kant Sahu K. Management of BK virus-associated haemorrhagic cystitis in allogeneic stem cell transplant recipients. Ther Adv Infect Dis. 2021;3(8):2049936121991377.
Infection and Drug Resistance

\section{Publish your work in this journal}

Infection and Drug Resistance is an international, peer-reviewed openaccess journal that focuses on the optimal treatment of infection (bacterial, fungal and viral) and the development and institution of preventive strategies to minimize the development and spread of resistance. The journal is specifically concerned with the epidemiology of antibiotic resistance and the mechanisms of resistance development and diffusion in both hospitals and the community. The manuscript management system is completely online and includes a very quick and fair peerreview system, which is all easy to use. Visit http://www.dovepress.com/ testimonials.php to read real quotes from published authors. 